\title{
Continents francophones, sous la direction de Sabrina Parent, Anne Douaire-Banny et Romuald Fonkoua
}

\section{Carminella Biondi}

\section{(2) OpenEdition}

\section{Journals}

\section{Edizione digitale}

URL: http://journals.openedition.org/studifrancesi/1484

DOI: $10.4000 /$ studifrancesi. 1484

ISSN: 2421-5856

Editore

Rosenberg \& Sellier

\section{Edizione cartacea}

Data di pubblicazione: 1 décembre 2015

Paginazione: 638-639

ISSN: 0039-2944

\section{Notizia bibliografica digitale}

Carminella Biondi, «Continents francophones, sous la direction de Sabrina Parent, Anne Douaire-Banny et Romuald Fonkoua », Studi Francesi [Online], 177 (LIX | III) | 2015, online dal 01 décembre 2015, consultato il 09 janvier 2021. URL: http://journals.openedition.org/studifrancesi/1484 ; DOI: https:// doi.org/10.4000/studifrancesi. 1484

Questo documento è stato generato automaticamente il 9 janvier 2021.

\section{(c) $(1) \odot$}

Studi Francesi è distribuita con Licenza Creative Commons Attribuzione - Non commerciale - Non opere derivate 4.0 Internazionale. 


\title{
Continents francophones, sous la direction de Sabrina Parent, Anne Douaire-Banny et Romuald Fonkoua
}

\author{
Carminella Biondi
}

\section{NOTIZIA}

«ElFe $\mathrm{XX}^{\mathrm{e}}$ et $\mathrm{XXI}^{\mathrm{e}}$ siècles», Continents francophones, sous la direction de Sabrina PARENT, Anne DOUAIRE-BANNY et Romuald FONKOUA, 2014, n. 4, pp. 187.

1 Per il dossier riunito in questo numero della «Revue annuelle de la Société d'étude de la littérature de langue française des $\mathrm{xx}^{\mathrm{e}}$ et $\mathrm{xxI}^{\mathrm{e}}$ siècles» si è scelto un titolo, almeno apparentemente, abbastanza vago da risultare quasi banale, Continents francophones, ma se si fa attenzione all'intero frontespizio della rivista ci si rende conto che quell'etichetta così generica non deve essere stata scelta a caso, perché fa da perfetto contrappunto alla definizione "littérature de langue française", al singolare, scelta invece per indicare l'oggetto di studi della rivista e della Società che la pubblica. Il solo accostamento dei due titoli fa, infatti, subito esplodere le contraddizioni e le ambiguità che caratterizzano il mondo francofono e che hanno fatto tanto discutere in questi anni. Come si coniuga l'idea di una letteratura in lingua francese, che sembra presupporre un'unità, con la molteplicità legata all'immagine di "continenti francofoni"? Una delle aporie o almeno uno dei grandi interrogativi che fanno fatica a trovare risposte adeguate, nonostante i fiumi di inchiostro che hanno fatto versare. Qualche goccia è caduta anche nelle pagine di questo volume, ad esempio nell'intervista che lo conclude, fatta da Sabrina PARENT à Marc QUAGHEBEUR: «Francophone», le mot qu'il s'agit d'habiter enfin (pp. 171-181), in cui si recuperano in chiave decisamente positiva la tanto vituperata etichetta e le sue diverse declinazioni.

2 Se il titolo del dossier è vago, ma, come si è visto, perfettamente funzionale e dirompente nel suo rapporto simmetrico con quello della rivista, l'introduzione (Allers- 
retours théoriques et méthodologiques dans les continents francophones, pp. 9-13), firmata dai tre curatori, tutti noti studiosi dei "fenomeni" francofoni, precisa invece bene il contenuto molto più puntuale ed omogeneo del volume e le sue finalità: "Les littératures francophones invitent naturellement à repenser le "fait littéraire" - qu'il s'agisse de ses production, réception, diffusion, instutionnalisation ou historiographie hors du cadre strictement national. La discussion sur ce sujet est en pleine ébullition [...] C'est dans ce contexte stimulant que la revue [...] s'est interrogée sur les phénomènes de type "osmotique" - transferts et échanges - opérant entre champs littéraires français et francophones, du Nord comme du Sud. En engageant un dialogue entre littératures francophones (Nord/Sud), littérature française et théorie/ méthodologies, les contributeurs de ce numéro se sont livrés à un véritable décloisonnement des domaines et à un désenclavement des systèmes de pensée» (p. 9). A prima vista potrebbe sembrare una di quelle prefazioni un po' altisonanti cui seguono frutti spesso modesti, ma bisogna riconoscere che in questo caso il progetto di rottura è in buona misura rispettato, in una miscellanea che pur affrontando argomenti disparati, trova una sua unità nella reinvenzioni di vecchi canoni o nella rilettura comparativa di testi erroneamente canonizzati in formule che non reggono di fronte alle più approfondite conoscenze storiche $\mathrm{o}$ al cambiato contesto culturale. Per esempio, il primo saggio di Chloé CHAUDET, Le renouveau de l'engagement littéraire, de Sartre aux littératures francophones. L'exemple de "La Femme sans sépulture" d'Assia Djebar (pp.15-33), recupera in chiave positiva un concetto sartriano che sembrava ormai desueto nelle nostre letterature occidentali, quello di letteratura impegnata, per rimodularlo nella formula di «dénonciation d'un inacceptable», perfettamente funzionale in area francofona, ed in particolare maghrebina, come dimostra la sua applicazione al romanzo di Assia Djebar. Bisogna dire che in questo dossier le quotazioni di Sartre sono in rialzo, come conferma anche il saggio di Dominique COMBE, «Situation d"“Orphée noir": Sartre et Césaire» (pp. 133-141), che non solo riconosce a Sartre un ruolo chiave nella ricezione delle letterature "nere" di lingua francese, ma ne fa uno dei capostipiti degli studi postcoloniali: "Aujourd'hui encore la critique américaine continue à lire "Orphée noir" comme un texte fondateur - avec Les Damnés de la terre de Fanon et le Portrait du colonisé d'Albert Memmi, également préfacés par Sartre, qui demeure l'un des penseurs français de référence pour les études postcoloniales» (p. 141). Scendono invece le quotazioni di Gide, il cui Voyage au Congo (1927) viene ridimensionato sia per quanto riguarda la sua presa di posizione anticolonialista che la sopravvalutata attenzione ai popoli e ai luoghi che attraversa. Certo, non bisogna cedere alla tentazione di letture decontestualizzate, ma Robert FOTSING MANGOUA, nel suo saggio André Gide, l'anticolonialisme revisité (pp.93-110), dimostra, attraverso la sua analisi puntuale, come Gide non fosse anticolonialista, ma si sia limitato a criticare le disfunzioni e pertanto le negatività del sistema, e come sia in fondo rimasto chiuso nel suo mondo: «Il est apparu que Gide, en emportant en Afrique une bibliothèque volumineuse, s'enfermait dans sa propre culture au lieu de s'ouvrir à l'ailleurs [...] sa représentation du continent noir reste conforme aux préjugés racistes élaborés par l'Occident depuis les années d'esclavage» (p. 109). Letto dall'altra sponda e in contesto postcoloniale il saggio di Gide mostra le corde. Ma non bisogna dimenticare che, all'epoca in cui è apparso, ha svolto un'innegabile funzione di critica del sistema coloniale o almeno di un modello di colonizzazione finalizzata al solo sfruttamento dei popoli colonizzati. 
Il secondo saggio del dossier, di Anthony MANGEON, intitolato Crimes d'auteur. Ou comment lire les littératures françaises et francophones façon série noire (pp. 35-67), non riguarda, o solo marginalmente, il romanzo poliziesco, perché i crimini di cui si tratta in questo saggio, crimini reali, non concernono una vittima qualunque, ma lo scrittore, evacuato dall'opera attraverso l'uso di uno pseudonimo, come è il caso di Romain Gary/Émile Ajar in Pseudo (1976), o attraverso lo scontro fra autore, narratore e personaggio che si disputano la paternità della storia in Banal oubli (2008) dello scrittore haitiano Victor Gary. Particolarmente interessante, in questo saggio, tutta la parte introduttiva in cui, attorno alla tematica indicata, quanto mai attuale in ambito postcoloniale, emergono molti dei problemi che concernono il dibattito attorno alle letterature di lingua francese. Segue un saggio che si sofferma su alcuni aspetti dello specifico letterario quebecchese, a partire dal concetto di "hétérolinguisme" creato da Rainier Grutman agli inizi degli anni '90 (Myriam sUCHET, De l'imaginaire hétérolingue à la lecture indisciplinaire. Propositions théoriques et méthodologiques à partir de quelques textes écrits en «québé-quois», pp.69-91), ma per allargarne poi la portata al più vasto ambito della letteratura, attraverso la teorizzazione, non so quanto convincente, di una «lecture indisciplinaire».

Completano il volume due lavori molto importanti sul piano del dibattito teorico e, per certi versi, complementari: Maxime DEL FIOL, Les "occidentalismes" de la littérature arabomusulmane d'expressions arabe et française depuis l'expédition d'Égypte. Pour une problématisation et une acclimatation du concept en critique littéraire (pp.111-132), e Guillaume BRIDET, Pour une histoire littéraire écrite du point de vue cosmopolite. Éléments à partir de la circulation de l'œuvre de Tagore à travers le monde (pp. 143-169). Entrambi, avendo come punto di riferimento l'opera di Edward Said, si interrogano sulla lettura speculare dell'Occidente da parte dell'Oriente e viceversa, tentando di andare oltre le barriere geografiche, linguistiche, ideologiche, senza però cadere nella trappola di un «mondial décontextualisé», senza cioè perdere il contatto con il reale: «saisir les textes littéraires au plus près de leur singularité comme également les auteurs dans leur plus grande autonomie: faits de ci et de ça, insoumis peut-être, certainement ouverts sur ce qu'on n'attendait pas» (p. 169).

5 Un numero davvero importante di questa giovane rivista, che cerca di rinnovare il dibattito teorico/metodologico sulle letterature di lingua francese e, più in generale, sulle letterature postcoloniali, fondandosi però sempre, rigorosamente, sulle opere, gli autori e il loro contesto di riferimento. 